\title{
Human $\beta$-defensin-2 production from S. cerevisiae using the repressible MET17 promoter
}

\author{
Thea S. B. Møller ${ }^{1,2}$, Joanna Hay ${ }^{1}$, Malcolm J. Saxton ${ }^{1}$, Karen Bunting ${ }^{1}$, Evamaria I. Petersen², Søren Kjærulff ${ }^{1}$ \\ and Christopher J. A. Finnis ${ }^{1 *}$
}

\begin{abstract}
Background: Baker's yeast Saccharomyces cerevisiae is a proven host for the commercial production of recombinant biopharmaceutical proteins. For the manufacture of heterologous proteins with activities deleterious to the host it can be desirable to minimise production during the growth phase and induce production late in the exponential phase. Protein expression by regulated promoter systems offers the possibility of improving productivity in this way by separating the recombinant protein production phase from the yeast growth phase. Commonly used inducible promoters do not always offer convenient solutions for industrial scale biopharmaceutical production with engineered yeast systems.

Results: Here we show improved secretion of the antimicrobial protein, human $\beta$-defensin-2, (hBD2), using the S. cerevisiae MET17 promoter by repressing expression during the growth phase. In shake flask culture, a higher final concentration of human $\beta$-defensin-2 was obtained using the repressible MET17 promoter system than when using the strong constitutive promoter from proteinase B (PRB1) in a yeast strain developed for high-level commercial production of recombinant proteins. Furthermore, this was achieved in under half the time using the MET17 promoter compared to the PRB1 promoter. Cell density, plasmid copy-number, transcript level and protein concentration in the culture supernatant were used to study the effects of different initial methionine concentrations in the culture media for the production of human $\beta$-defensin- 2 secreted from S. cerevisiae.
\end{abstract}

Conclusions: The repressible S. cerevisiae MET17 promoter was more efficient than a strong constitutive promoter for the production of human $\beta$-defensin-2 from S. cerevisiae in small-scale culture and offers advantages for the commercial production of this and other heterologous proteins which are deleterious to the host organism. Furthermore, the MET17 promoter activity can be modulated by methionine alone, which has a safety profile applicable to biopharmaceutical manufacturing.

Keywords: MET17 promoter, Saccharomyces cerevisiae, Yeast, Human $\beta$-defensin-2

\section{Background}

Saccharomyces cerevisiae has a long safe history of use for the production of biopharmaceutical proteins and has a rich density of knowledge detailing its genetics, biochemistry, physiology and large-scale fermentation performance. Many different promoters have been used to successfully drive the expression of foreign genes in S. cerevisiae. However, the choice of promoter for

\footnotetext{
*Correspondence: chrisfinnis@hotmail.com

1 Novozymes Biopharma UK Limited, Castle Court, 59 Castle Boulevard, Nottingham NG7 1FD, UK

Full list of author information is available at the end of the article
}

heterologous gene expression can affect the product yield greatly $[1,2]$. Expression using regulated promoter systems is advantageous for proteins which are toxic to the host cell as this allows controlled timing of gene expression and higher production levels. Regulated expression can be achieved through manipulation of the growth medium by adding metabolites. The galactose (GAL110) promoters are commonly used to allow regulation of the target gene by the carbon source; using galactose for induction and glucose for repression [3]. However, the commonly used galactose promoter systems are not always applicable for industrial expression systems, as 
the $S$. cerevisiae strains used may not respond well to galactose. In addition, regulation of these promoters may interfere with the cellular metabolism and in many cases the regulation is not tight enough to completely shut off transcription. This issue was addressed by use of the tetracycline (Tet-On/Off) promoters, which are either inducible or repressible [4]. Here, gene expression is activated as a result of binding of the Tet-Off or Tet-On protein to an element located within an inducible promoter. One advantage of this system is that promoter regulation with the tetracycline derivative, doxycycline does not interfere with the yeast cellular metabolism. However, doxycycline is not ideal for use in biopharmaceutical processes and its regulation needs tetracycline-regulated activators and repressors, which require a specific strain background or additional manipulations of the strains in use $[4,5]$. Consequently, alternative promoter systems with safe and simple regulation are desirable for some large scale biopharmaceutical processes.

In S. cerevisiae, the MET17 gene (also known as MET15 and $M E T 25)$ encodes $O$-acetylhomoserine sulfhydrolase $[6,7]$ and its promoter displays repression of transcriptional expression in the presence of methionine [8] or $S$-adenosylmethionine (SAM) [9, 10]. MET17 catalyses the last step of the sulfate assimilation pathway in S. cerevisiae, which is the incorporation of sulfide into a carbon chain [7]. The promoter is efficiently and strongly repressed at high methionine concentrations with the expression of $\mathrm{O}$-acetylhomoserine sulfhydrolase only occurring below $0.05 \mathrm{mM}$ methionine $[6,7,11]$. Utilising media with the correct concentration of methionine, the MET17 promoter has been used previously to express human serum albumin (HSA) and albumin fusion proteins, including repressing production of glucagon-HSA in early log phase with expression in late log phase [6]. This separation of the growth and production phases may be especially useful for expressing proteins that are toxic to the yeast host, which are best produced in the late log phase. However, a separation in growth and production phases cannot be achieved using constitutive promoters, where the secretion of toxic, or even relatively nontoxic proteins, can be deleterious to the host resulting in reduced product yields. Here, the adverse effects of the recombinant hBD2 on the host may be either intracellular during secretion, or extracellular due to its accumulation to toxic levels in the growth media. Compared to inducible systems such as the galactose system, the MET17 promoter does not require a change in carbon source that may potentially slow growth, or the addition of an inducing metabolite [6]. This system is based upon the consumption of methionine from the media leading to subsequent expression of the gene of interest downstream of the MET17 promoter. In the past, the MET17 promoter has been used to produce several heterologous proteins under derepressing conditions, including $\beta$-galactosidase [12], CaArn1, a siderophore transporter from Candida albicans [13], green fluorescent protein (GFP) and GFP fusions [14], and human albumin and albumin fusions [6].

Here we describe the use of the repressible MET17 promoter for the secretion of human $\beta$-defensin-2 in shake flask cultures (SFC) using a highly productive S. cerevisiae strain. This yeast strain was initially developed for the secretion of recombinant human albumin (rHA); however, studies have shown that it can also be used to express a diverse range of heterologous proteins [15]. $\mathrm{hBD} 2$ belongs to the intriguing class of antimicrobial and immunomodulatory peptides called defensins. Defensins are small cationic and cysteine-rich peptides that play a crucial role in the host defence against microorganisms $[16,17]$. hBD2 is a 41 amino acid peptide first characterised in psoriatic skin, which has been shown to be active against Gram-negative (E. coli, $P$. aeruginosa) and Gram-positive ( $S$. aureus) bacteria as well as yeast $(C$. albicans, C. krusei, C. parapsilosis) [18-20]. The action of defensins against microorganisms generally involves membranolytic disruption, permeability, or pore formation [21]. Their amino acid composition, amphipathicity, cationic charge and size allow them to attach to and insert into membrane bilayers to form pores [22].

Heterologous expression systems have the potential to provide hBD2 for both clinical research and therapeutic applications. However, the issues of host cell toxicity, proteolytic degradation, folding and low yields must be overcome to provide a suitable platform for commercial production. To date, recombinant hBD2 has predominantly been produced from inducible $E$. coli expression systems as a fusion protein requiring in vitro cleavage [23]. Subsequent oxidation may also be needed, for which yields of 1-2 mg final product per litre of bacterial culture have been reported [24]. However, soluble active hBD2 can also be obtained from fusion proteins without the need for refolding, where yields estimated to be around $100 \mathrm{mg} / \mathrm{L}$ purified mature $\mathrm{hBD} 2$ have been obtained after enterokinase cleavage [25]. In contrast, S. cerevisiae offers the advantage of secreting fully folded mature bioactive hBD2 into the culture medium at relatively high product titres without the need for costly in vitro processing.

Here we report that the repressible MET17 promoter produced the highest levels of hBD2 under initially repressing conditions, which was achieved in half the incubation time compared to the strong constitutive PRB1 promoter in a yeast system modified for high-level production of biopharmaceutical proteins. Normally, the $P R B 1$ promoter is regulated by carbon and nitrogen 
sources as well as growth phase, which results in a transcriptional derepression of the PRB1 promoter as the cells approach the stationary phase of growth [26]. However random mutagenesis of this proprietary yeast strain produced a constitutive $P R B 1$ promoter system, which allows expression of the target protein during exponential growth as well as during late exponential growth [27].

\section{Results}

High-level expression of human $\beta$-defensin- 2 from the MET17 promoter is induced by methionine depletion

To determine if the repressible MET17 promoter was more efficient for hBD2 expression than the strong constitutive $P R B 1$ promoter, expression plasmids were produced harbouring the gene for hBD2 downstream of either the MET17 or PRB1 promoter. An expression plasmid comprising the MET17 promoter upstream of the rHA gene was also made to compare the expression of a non-toxic protein to that of a toxic protein. A low productivity $S$. cerevisiae strain, DB1 and a high productivity $S$. cerevisiae strain, DYB7, were used for these studies (Table 1).

Shake flask cultures of yeast strains were grown in non-repressing (methionine-free medium) and repressing (1000 $\mu \mathrm{M}$ initial methionine concentration) conditions to control protein production. Analysis of the SFC supernatants of DYB7 [pDB3936:GR:pDB4351], expressing hBD2 from the MET17 promoter, by SDSPAGE (Fig. 1) and ultra-performance liquid chromatography mass spectrometry (UPLC-MS) (Fig. 2) revealed that the hBD2 expression was significantly improved by initial repression of the MET17 promoter. Results for the PRB1 promoter driven constructs confirmed that derepression only occurred with the MET17 promoter constructs. The UPLC-MS data indicated that a higher hBD2 concentration was obtained in DYB7 using the MET17 promoter repressed by $1000 \mu \mathrm{M}$ methionine compared to the $P R B 1$ promoter in less than half the incubation time (Fig. 2a, b). The hBD2 yield using the MET17 promoter was estimated to be approximately double that of the $P R B 1$ promoter after $70 \mathrm{~h}$.

Table 1 Yeast strains used in this work

\begin{tabular}{lll}
\hline Strain & Known genotype & Reference \\
\hline DB1 & cir $^{0}$, MATa, leu2-3, leu2-112 & {$[27]$} \\
DBY7 & rHA-overproducing strain derived from DB1 by & {$[39]$} \\
& $\begin{array}{c}\text { chemical mutagenesis. cir }{ }^{0}, \text { MATa, leu2-3, leu2-112, } \\
\\
\end{array}$ & \\
\hline
\end{tabular}

This demonstrated that the expression of antimicrobial defensin hBD2 from the repressible MET17 promoter was advantageous and resulted in a higher overall yield (g/L/h), an important factor for the economics of biopharmaceutical manufacturing.

In order to demonstrate the effect of strain optimisation from successive rounds of chemical mutagenesis and selection, production of hBD2 by the MET17 promoter was also analysed in the original progenitor strain DB1 and compared to a mutated high-level production strain DYB7 [27-30]. In SFC, DYB7 showed an approximate four-fold increase in hBD2 production levels compared to the original progenitor strain DB1, under initially repressing conditions (Fig. 2a, c). Furthermore, the benefits of repressing hBD2 expression early in the growth phase were greater in the higher productivity strain DYB7.

Recombinant human albumin (rHA) expression from the MET17 promoter was used as a control for expression of a non-toxic protein compared to hBD2 (Fig. 2d). Similar rHA protein concentrations were obtained after $120 \mathrm{~h}$ of expression regardless of whether the MET17 promoter was repressed or not in DYB7. Therefore, this UPLC-MS data indicates that the MET17 promoter is a valuable tool for the production of toxic proteins in this expression system.

\section{High hBD2 mRNA levels were obtained from a derepressed MET17 promoter}

Derepression of the MET17 promoter was demonstrated in quantitative RNA studies from both $S$. cerevisiae DB1 and DYB7 by marked increases in the hBD2 mRNA levels during the exponential growth phase when methionine was initially present in the media (Fig. 3a, c). This increase in mRNA was likely to be caused by the derepression of the MET17 promoter as it was induced by methionine depletion during cell growth. It was observed that the maximum hBD2 transcript levels from the MET17 promoter occurred earlier for DB1 than the higher productivity strain DYB7, which can be attributed to growth rate differences between these strains (see Fig. 5a, c). Significantly lower transcript levels were seen in the cultures without methionine in the media, which indicated that a higher overall mRNA level was obtained from an initially repressed MET17 promoter. The transcript levels from the $P R B 1$ promoter-driven construct confirmed that derepression was specific to the MET17 promoter constructs, as the $P R B 1$ promoter was not affected by the methionine concentrations used in this study. Changes in the transcript levels throughout the expression study are 


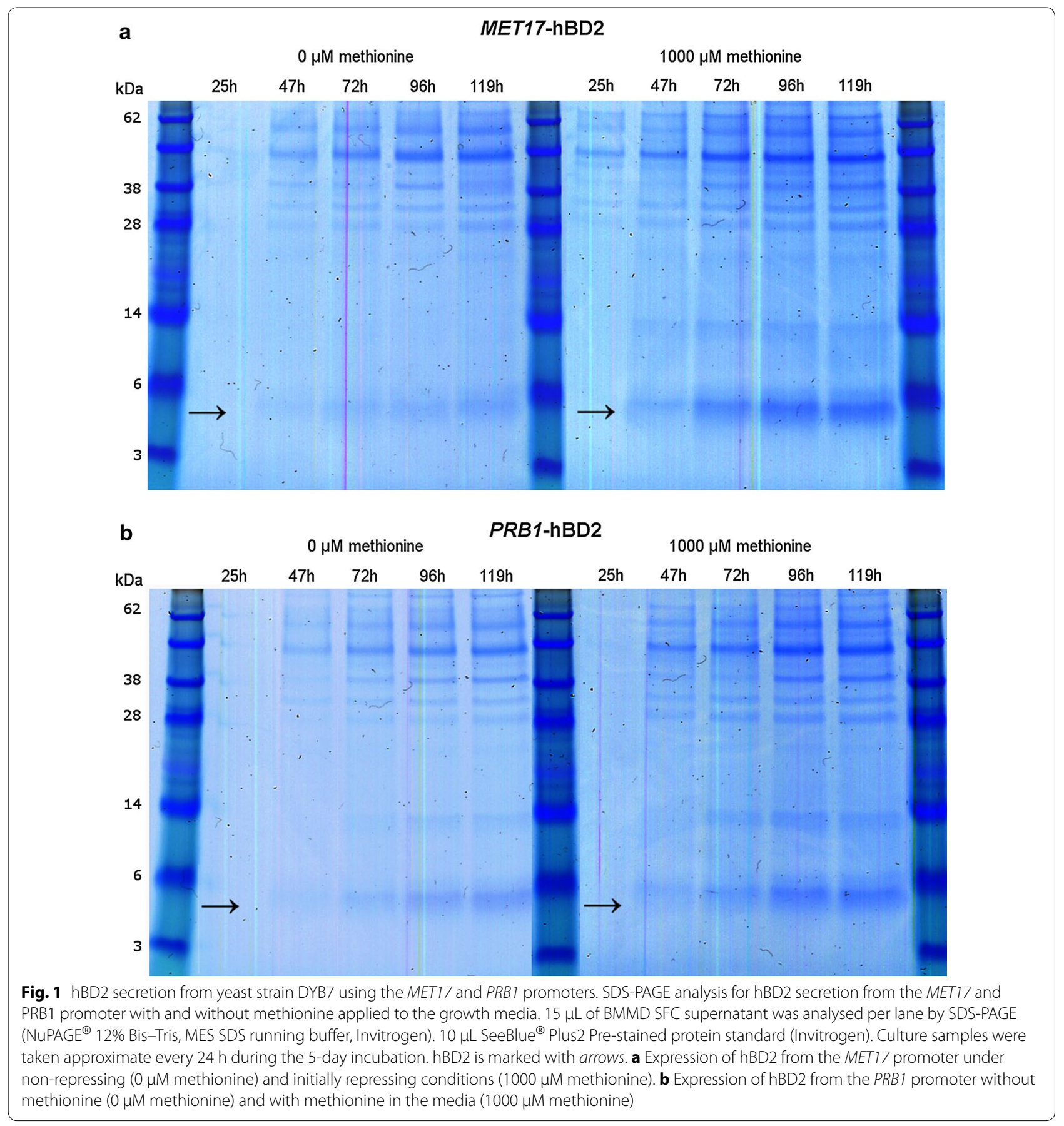

attributed to changing environmental conditions during these batch cultures.

A verification of two endogenous controls was performed to demonstrate which one was the most suitable endogenous control for the type of assay performed (results not shown). ACT1 was chosen due to its history of frequent use as an endogenous control in RT-PCR experiments [31, 32]. TAF10 (TATA binding protein associated factor) was selected based on the research by Teste et al. which demonstrated that it is a suitable endogenous control because its expression was the most stable under the conditions tested. The transcript levels of these genes were analysed by a direct comparison of their cycle threshold $\left(C_{t}\right)$, assuming equal $C_{t}$ for equal 

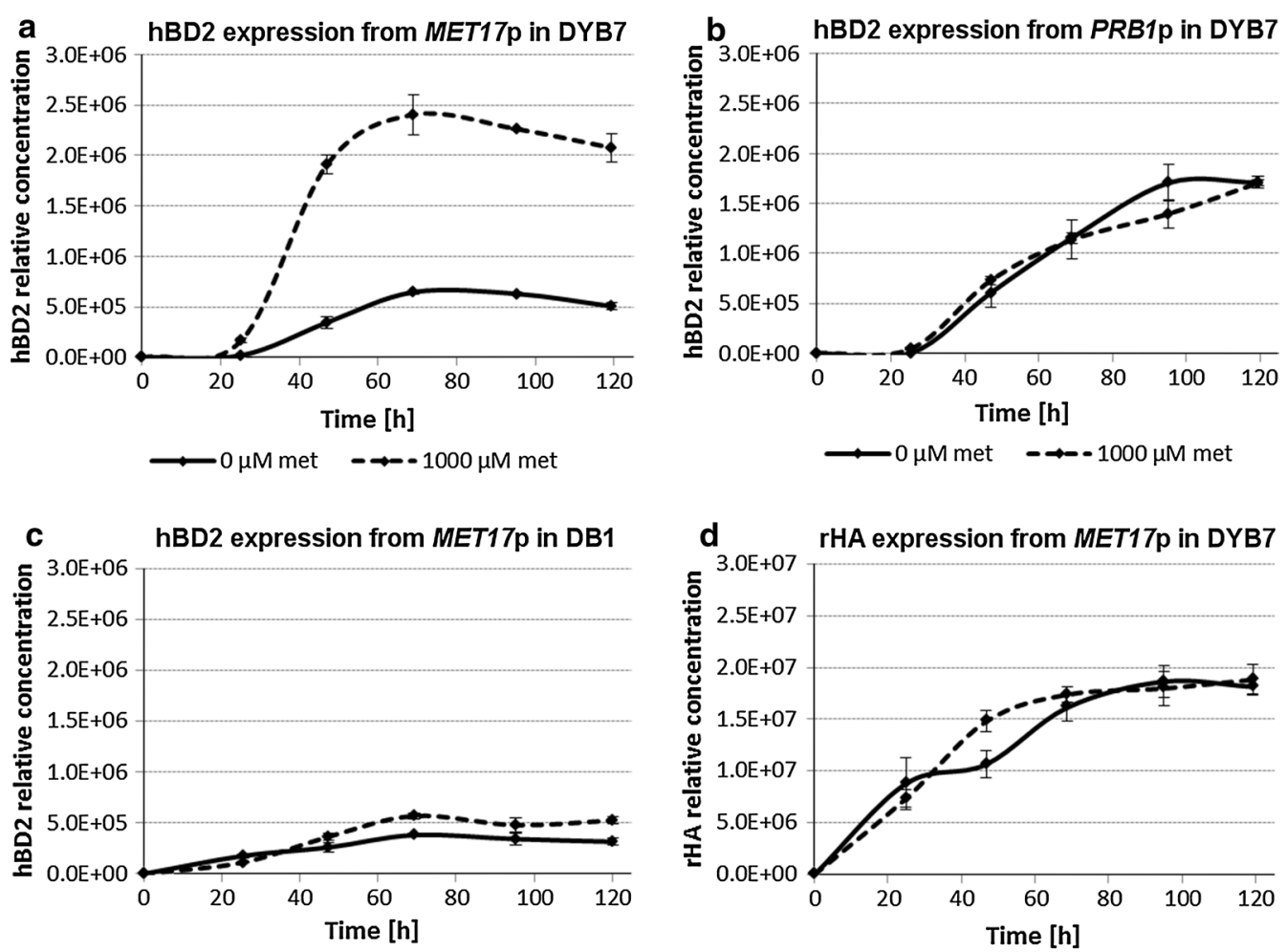

$\longrightarrow 0 \mu \mathrm{M}$ met $\quad-\rightarrow-1000 \mu \mathrm{M}$ met

$\longrightarrow 0 \mu \mathrm{M}$ met $\quad-\rightarrow-1000 \mu \mathrm{M}$ met

Fig. 2 Relative hBD2 and rHA productivity determined by UPLC-MS analysis. hBD2 and rHA were expressed using either the MET17 or the PRB1 promoter on $2 \mu \mathrm{m}$-based expression plasmids. Plasmids were transformed into $S$. cerevisiae strain DYB7 or DB1. The transformed yeast were inoculated at $\mathrm{OD}_{600}=0.15$ into BMMD SFC without or with $1000 \mu \mathrm{M}$ methionine and grown for 5 days, while culture samples were taken approximately every $24 \mathrm{~h}$. Error bars indicate standard deviations $(\mathrm{n}=3$ ). a Expression of hBD2 from the MET17 promoter (pDB3936:GR:pDB4351) under nonrepressing ( $0 \mu \mathrm{M}$ methionine) and initially repressing conditions (1000 $\mu \mathrm{M}$ methionine) in DYB7. b Expression of hBD2 from the PRB1 promoter (pDB3936:GR:pDB4146) without methionine $(0 \mu \mathrm{M})$ and with methionine in the media initially (1000 $\mu \mathrm{M})$ in DYB7. c Expression of hBD2 from the MET17 promoter (pDB3936:GR:pDB4351) under non-repressing (0 $\mu \mathrm{M}$ methionine) and initially repressing conditions (1000 $\mu \mathrm{M}$ methionine) in DB1. d Expression of $\mathrm{rHA}$ from the MET17 promoter (pDB3936:GR:pDB4692) under non-repressing (0 $\mu \mathrm{M}$ methionine) and initially repressing conditions (1000 $\mathrm{\mu M}$ methionine) in DYB7

transcript level, since all RT-PCR reactions were performed with equal quantity of total RNA (cDNA). The $\mathrm{C}_{\mathrm{t}}$ values for TAF10 displayed a smaller variation and gradient than the $\mathrm{C}_{\mathrm{t}}$ values for $A C T 1$. Consequently, TAF10 appeared to be a better endogenous control than $A C T 1$ for the experiments performed in this study. Based on these results TAF10 was used as the endogenous control all RT-PCR experiments.

\section{Plasmid copy-number was affected by promoter activity}

Significantly higher relative plasmid copy-numbers were obtained in the high productivity strain DYB7, compared to the original progenitor strain DB1, which is consistent with the $u b c 4$ mutation in DYB7 causing an increased plasmid copy-number (Fig. 4) [28]. The results obtained through copy-number determination using (RT-PCR) did not show a significant difference between cultures with and without methionine repression in any strain.

\section{Higher cell densities in hBD2 cultures with a repressed MET17 promoter}

In small scale cultures, a higher biomass was achieved earlier in the exponential growth phase in the repressed cultures of both DB1 and DYB7 with hBD2 expression from the MET17 promoter (Fig. 5a, c). The repression effect was much more pronounced when the yeast cells were producing hBD2, compared to the albumin control (Fig. 5d), presumably because of the greater burden on host cell metabolism. The difference in growth between the cultures expressing rHA from the MET17 promoter and the cultures expressing hBD2 from the same promoter are consistent with the antimicrobial activity of 

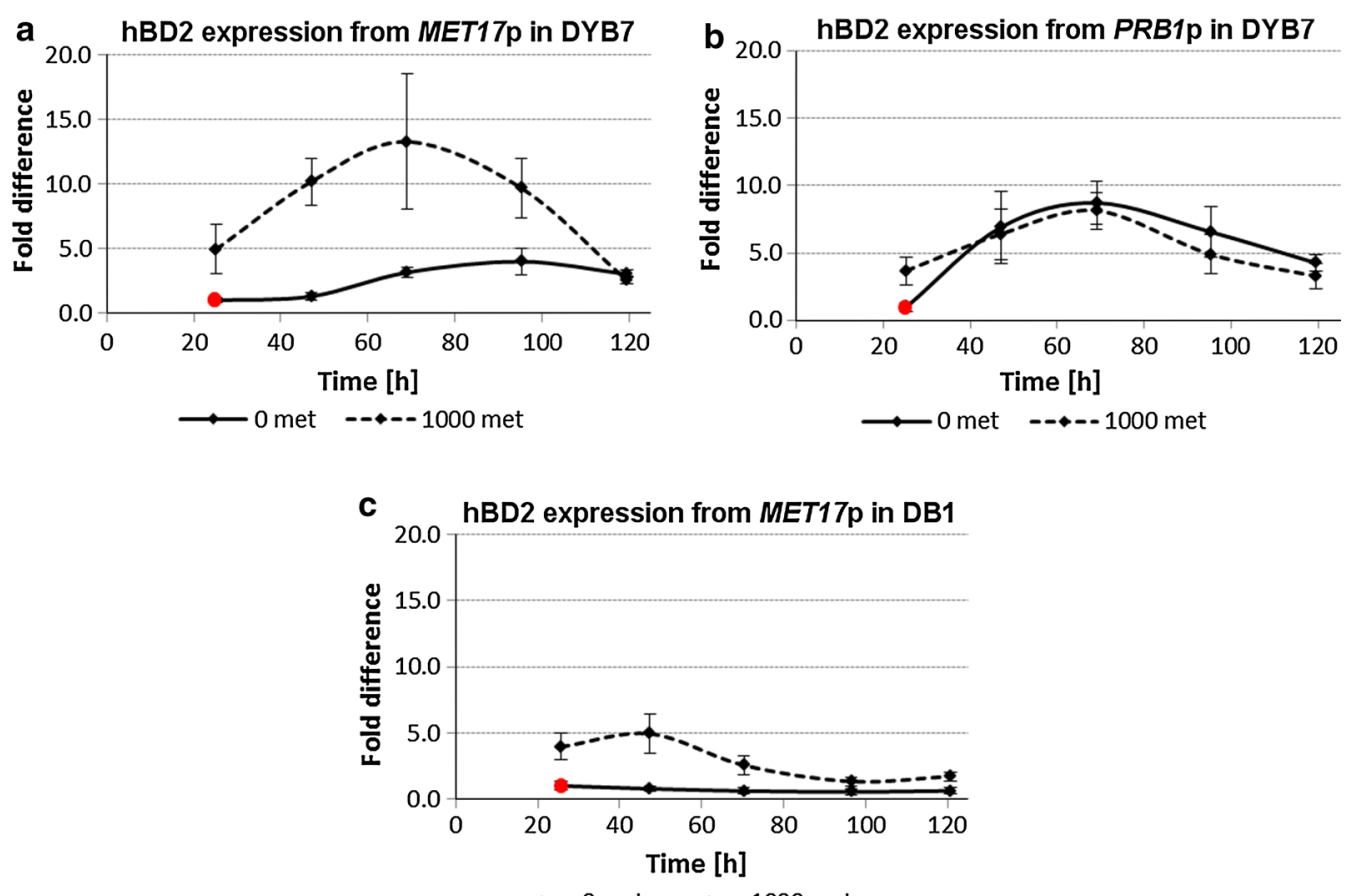

Fig. 3 Transcript levels determined by real-time PCR. hBD2 was expressed from a $2 \mu \mathrm{m}$-based expression plasmid containing either the MET17 or PRB1 promoter upstream of the hBD2 gene. These plasmids were transformed into $S$. cerevisiae strains DYB7 or DB1. The transformed yeast were inoculated at $\mathrm{OD}_{600}=0.15$ into BMMD SFC without or with $1000 \mu \mathrm{M}$ methionine and grown for 5 days, while cell pellet samples were taken approximately every $24 \mathrm{~h}$ and stored in RNAlater (Invitrogen) before RNA isolation and subsequently cDNA preparation. Error bars indicate coefficient of variation $(n=6)$. The fold difference is relative to the culture without methionine at $24 \mathrm{~h}$ in each strain (marked in red). a hBD2 mRNA produced from DYB7 with the MET17 promoter under non-repressing ( $0 \mu \mathrm{M}$ methionine) and repressing conditions (1000 $\mu \mathrm{M}$ methionine). b hBD2 mRNA produced from DYB7 with the PRB1 promoter without methionine added to the media $(0 \mu \mathrm{M})$ and with added methionine $(1000 \mu \mathrm{M})$. c hBD2 mRNA produced from DB1 with the MET17 promoter under non repressing (0 $\mu \mathrm{M}$ methionine) and repressing conditions (1000 $\mu \mathrm{M}$ methionine). TAF10 was used as the endogenous control

hBD2 inhibiting the cell growth. The PRB1 promoter driven constructs (Fig. 5b) confirmed that the repression effect was specific to the MET17 promoter in both low and high productivity $S$. cerevisiae strains. The antimicrobial effect of hBD2 was also observed on the growth curves of DYB7 when the PRB1 promoter was used compared to the MET17 promoter under initially repressing conditions, as a longer lag phase was observed (Fig. 5a, b). The growth inhibiting effect of $\mathrm{hBD} 2$ production was less marked for DB1 than for DYB7 (Fig. 5a, c), potentially due to the faster growth and lower productivity of DB1 compared to DYB7, suggesting that exceeding a critical hBD2 concentration for a given cell density may be required for cell growth to be inhibited.

Consequently, a higher biomass was obtained earlier using the MET17 promoter initially repressed by methionine, because a separation of the hBD2 production phase and the yeast growth phase had been achieved.

\section{Discussion}

For the cultures containing $1000 \mathrm{mM}$ methionine in the initial medium it is interesting that this does not appear to have contributed to a significant increase in growth rate compared to the cultures lacking this additional nutrient (Fig. 5b, d). Neither does it appear to have had a significant effect on the production of either hBD2 or rHA (Fig. 2b, d), suggesting it has not alleviated a limitation to their secretion. It is however, possible that overall protein expression might have been affected and background staining of SDS-PAGE gels appears to be greater for cultures with $1000 \mathrm{mM}$ methionine in the initial media (Fig. 1), which is otherwise a minimal medium with micronutrients supplemented, but devoid of amino acids. If so, this effect could add to the stimulatory effect of initial repression of hBD2 and further boost the production of the target protein. 


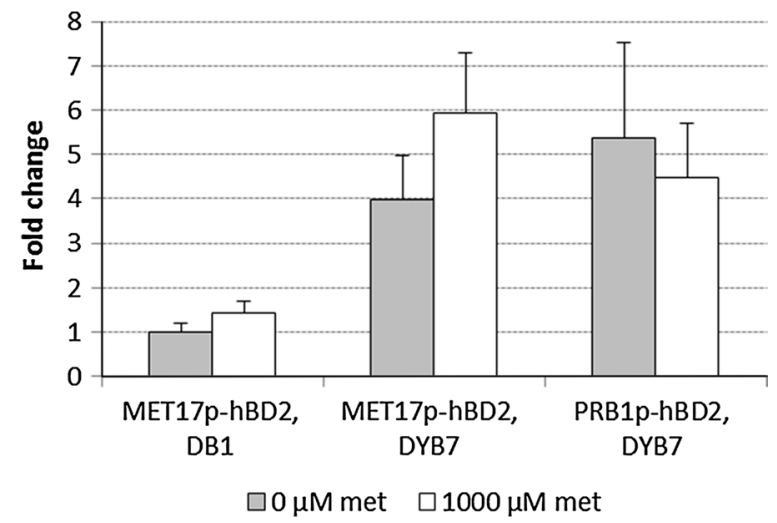

Fig. 4 Relative plasmid gene copy-number determined from realtime PCRs. The transformed yeast were inoculated at $\mathrm{OD}_{600}=0.15$ into BMMD SFC without or with $1000 \mu \mathrm{M}$ methionine and grown for $24 \mathrm{~h}$. The cells were harvested and total DNA was isolated from $10 \mathrm{~mL}$ cultures. The plasmid names refer to the gap-repair plasmids containing the expression cassettes with the promoter and the expressed protein indicated below, all of which are present in the final pSAC35based expression plasmid in yeast. Cultures were grown without and with $(1000 \mu \mathrm{M})$ methionine in the initial media. Error bars represent the standard deviation of triplicate analysis of triplicate experimental cultures $(n=9)$. The fold change is relative to DB1 [pDB4351] without methionine. FLP1 was used as the single-copy plasmid gene and TAF10 was used as the genomic control

It is interesting that the increase in hBD2 expression due to initial repression with methionine was so much greater for the higher productivity strain DYB7 compared to the progenitor strain DB1. This effect might be attributed to the greater toxicity caused by unregulated higher level hBD2 secretion, both intracellularly and also in the culture medium, where a threshold hBD2 concentration may be required for inhibitory effects on the host. While the hBD2 inhibition of Candida species is reported to vary considerably between strains in the range of $3.9 \mu \mathrm{g} /$ $\mathrm{mL}$ to $>250 \mu \mathrm{g} / \mathrm{mL}$ [21], the inhibitory concentration for DB1 and DYB7 remains to be defined, and indeed may differ due to the mutagenesis perform on DYB7.

Due to the low biomass available at early time points in this study, the first transcript data were at $24 \mathrm{~h}$, where hBD2 mRNA levels already appeared to be substantially higher when methionine was initially present in the culture media (Fig. 3a, c). Indeed, for both DYB7 and DB1 the hBD2 transcript levels are higher for the initially repressed cultures over the entire time course, suggesting that they had overshot the levels in the non-expressed cultures before $24 \mathrm{~h}$. It is possible that by this time the increased biomass shown in Fig. 5a, c for the initially repressed cultures might alleviate the toxic effects on the culture during the next $24 \mathrm{~h}$ period when the majority of hBD2 was produced (Fig. 2a).
Although it is clear from Fig. 4 that DYB7 has a higher plasmid copy number than DB1, the differences between repressed and non-repressed cultures with hBD2 expressed from the MET17 promoter are not significant in this study. Further analysis is therefore required to establish whether $M E T 17$ promoter transcription impacts on the transcription of the REP1, REP2, FLP1 and $D$ genes, which might affect the plasmid copy-number through their roles controlling plasmid amplification and partitioning between mother and daughter cells [33, 34].

While accurate quantification of the mature hBD2 expression levels has not been performed in this study, the intensity of bands observed by SDS-PAGE suggests that high-levels of hBD2 production might be achieved during large scale fermentation using this well characterised yeast system. Due to the inherent scalability of this yeast system and its extensive industrial use for the production of human albumin and other recombinant proteins, expression levels of several hundred milligrams per litre of this $4.3 \mathrm{KDa}$ protein might be expected, based on the equivalent molar expression levels of other proteins produced at large scale. Furthermore, the encouraging results described here, suggest that optimisation of methionine concentration during high-cell density fermentation could be used to maximise hBD2 yields when using the MET17 promoter for large scale production. It would also be interesting to know at which time point the methionine is depleted and to perform metabolomics studies of the intracellular amino acid pools. Overall, the successful track-record of this yeast expression platform for the production of approved biopharmaceutical proteins and the economic benefits of secreting active soluble hBD2, without the need for expensive in vitro processing, make it an attractive candidate for further studies to be performed at large scale [35].

\section{Conclusions}

Improved production of hBD2 was obtained in shake flask culture using the repressible MET17 promoter under initially repressing conditions. Higher final concentrations of hBD2 were obtained using the repressible MET17 promoter compared to the strong constitutive PRB1 promoter in a S. cerevisiae strain developed for the high-level commercial production of recombinant proteins such as human albumin. Furthermore, the MET17 promoter system gave a higher $\mathrm{hBD} 2$ concentration in less than half the time of the PRB1 promoter system in this industrial yeast system. It was also shown that protein production could be controlled by the MET17 promoter through addition of methionine to the media, which was advantageous for the production of hBD2 and 

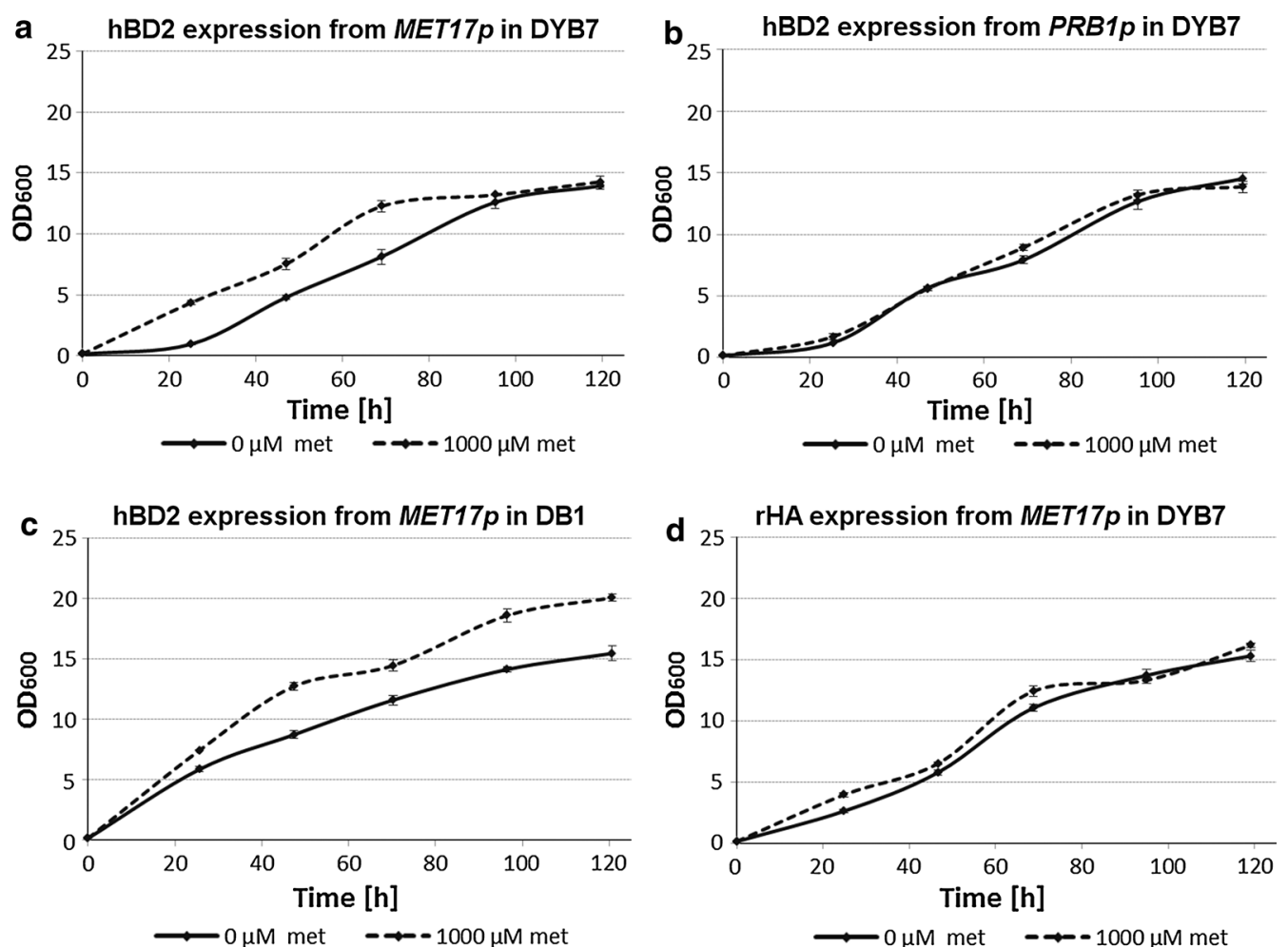

Fig. 5 Growth of DYB7 and DB1 producing hBD2 or rHA. rHA and hBD2 were expressed using $2 \mu \mathrm{m}$ expression plasmids containing either the MET17 or PRB1 promoter upstream of the coding regions. These plasmids were transformed into S. cerevisiae strain DB1 or DYB7. The transformed yeast were inoculated at $\mathrm{OD}_{600}=0.15$ into BMMD SFC with or without $1000 \mu \mathrm{M}$ methionine and grown for 5 days, while $\mathrm{OD}_{600}$ measurements were done approximately every $24 \mathrm{~h}$. Error bars indicate standard deviations $(n=3)$. a Growth of DYB7 [pDB3936:GR:pDB4351], which express hBD2 from the MET17 promoter under non-repressing (0 $\mu \mathrm{M}$ methionine) and initially repressing conditions (1000 $\mu \mathrm{M}$ methionine). b Growth of DYB7 [pDB3936:GR:pDB4146], which express hBD2 from the PRB1 promoter without methionine (0 $\mu$ M methionine) and with methionine in the media initially (1000 $\mu$ M methionine). c Growth of DB1 [pDB3936:GR:pDB4351], which express hBD2 from the MET17 promoter under non-repressing (0 $\mu \mathrm{M}$ methionine) and initially repressing conditions (1000 $\mu \mathrm{M}$ methionine). d Growth of DYB7 [pDB3936:GR:pDB4692], which express rHA from the MET17 promoter under non-repressing ( $0 \mu \mathrm{M}$ methionine) and initially repressing conditions (1000 $\mu \mathrm{M}$ methionine)

may also be beneficial for other proteins that are deleterious to the host. Overall, these results showed great potential to using the MET17 promoter for optimising production of difficult proteins from S. cerevisiae, which is likely to be applicable to large scale manufacturing of biopharmaceutical proteins.

\section{Methods}

\section{Plasmids and yeast strains}

Plasmid pDB4081 contains the PRB1 promoter, a secretory leader sequence, the coding sequence for rHA and the $S$. cerevisiae $A D H 1$ transcription terminator. The DNA sequence of the $P R B 1$ promoter is -819 to $-1 \mathrm{bps}$ from the start codon of the PRB1 gene on Chr. V 4004641953 [GeneID:856649]. The DNA sequence for rHA is a human albumin sequence [GeneID:213] codon optimised for expression in S. cerevisiae. The $5^{\prime}$-end of the human albumin DNA was altered to replace the native secretory leader sequence with a modified version of the pre-pro
HSA $/ M F \alpha 1$ fusion leader sequence [30] consisting of the amino acid sequence MKWVFIVSILFLFSSAYSRSLDKR (referred to as mHSA/MF 1 -leader) [18]. Additionally, the $3^{\prime}$-end of the human albumin DNA was modified to introduce two TAA stop codons. pDB4692 was constructed by cloning the MET17 promoter in place of the PRB1 promoter in pDB4081. The DNA sequence of the MET17 promoter is -388 to -1 bps from the start codon of the MET17 gene on Chr. VII 732542-733876 [GeneID:851010]. The hBD2 DNA fragment was cloned between the PRB1 promoter and the $A D H 1$ terminator in pDB4081 to replace the rHA gene and create pDB4146. The human $\beta$-defensin-2 sequence on Chr. 8 7752099-7754237 was codon optimised for expression in S. cerevisiae and an additional TAA stop codon was applied to the sequence. pDB4351 was constructed by cloning the MET17 promoter into $\mathrm{pDB} 4146$ in place of the PRB1 promoter. The MET17 and PRB1 promoters in pDB4146 and pDB4351 are linked to the $M F \alpha 1$ 
(mating factor alpha) leader sequence (MRFPSIFTAVLFAASSALAAPVNTTTEDETAQIPAEAVIGYLDLEGDFDVAVLPFSNSTNNGLLFINTTIASIAAKEEGVSLD, [GeneID:296148471]) fused to the plectasin pro sequence plus a Kex2 protease cleavage site, with the sequence APQPVPEAYAVSDPEAHPDDFAGMDANQLQKR. These expression cassettes were cloned into a $2 \mu \mathrm{m}$ plasmid, pDB3936 derived from of pSAC35 [36] and transformed into $S$. cerevisiae DB1 and DYB7 simultaneously for gap-repair recombination [37, 38]. In pDB3936, the LEU2 gene of pSAC35 has been truncated by replacing KpnI-NotI fragment with a synthetic linker, resulting in removal of the NotI site. Plasmids pDB4081, pDB4146, pDB4351 and pDB4692 contain the rHA or hBD2 expression constructs flanked by DNA for homologous recombination with pDB3936. Gap-repair transformants harbouring yeast expression disintegration plasmids were obtained by introducing purified Acc65I-BamHI "gapped plasmid" DNA (pDB3936) and purified PvuI-NsiI "insert" DNA. Plasmids obtained through gap-repair were named on basis of the origin of the "gapped plasmid" (pDB3936) and the plasmid of the "insert" fragment (e.g. pDB4692) using the convention pDB3936:GR:pDB4692. The yeast strains and expression plasmids used in this study are listed in Tables 1 and 2, respectively.

\section{Culture conditions}

Shake flask cultures with $10 \mathrm{~mL}$ BMMD (buffered minimal media dextrose [28]) were grown in $50 \mathrm{~mL}$ conical shake flasks to an $\mathrm{OD}_{600}$ of $1.5-2.5$ at $30{ }^{\circ} \mathrm{C}, 200 \mathrm{rpm}$, $25 \mathrm{~mm}$ orbit. $670 \mu \mathrm{M}$ methionine was used in the hBD2 expressing pre-cultures to repress $\mathrm{hBD} 2$ expression. The amount of this pre-culture required to inoculate the main culture at $\mathrm{OD}_{600}$ of 0.15 was calculated, pelleted by centrifugation and washed with BMMD medium to eliminate any methionine used in the pre-culture. This washed

\section{Table 2 Description of plasmids used in this work}

\begin{tabular}{|c|c|}
\hline Plasmid & Description \\
\hline pDB3936 & $\begin{array}{l}\text { pSAC35, a } 2 \mu \mathrm{m} \text {-derived yeast episomal expression vector } \\
\text { with a truncated LEU2 [36] }\end{array}$ \\
\hline pDB4081 & $\begin{array}{l}\text { Containing the expression cassette; } P R B 1 \text { promoter, the } \\
\text { mHSA/MFa1-leader leader, the coding sequence of rHA } \\
\text { and the S. cerevisiae ADH1 transcription terminator }\end{array}$ \\
\hline pDB4146 & $\begin{array}{l}\text { Containing the expression cassette; PRB1 promoter, the } \\
\text { MFa1/plectasin leader, the coding sequence of hBD2 } \\
\text { and the S. cerevisiae ADH1 transcription terminator }\end{array}$ \\
\hline pDB4351 & $\begin{array}{l}\text { Containing the expression cassette; MET17 promoter, the } \\
\text { MFa1/plectasin leader, the coding sequence of hBD2 } \\
\text { and the S. cerevisiae ADH1 transcription terminator }\end{array}$ \\
\hline pDB4692 & $\begin{array}{l}\text { Containing the expression cassette; MET17 promoter, the } \\
\text { mHSA/MFa1-leader leader, the coding sequence of } \mathrm{rHA} \\
\text { and the S. cerevisiae ADH1 transcription terminator }\end{array}$ \\
\hline
\end{tabular}

cell pellet was then resuspended in the appropriate volume of BMMD with the correct concentration of methionine and grown for 5 days at $30{ }^{\circ} \mathrm{C}, 200 \mathrm{rpm}$ in $50 \mathrm{~mL}$ conical shake flasks.

\section{SDS-polyacrylamide gel electrophoresis (PAGE)}

Culture supernatant was run on NuPAGE ${ }^{\circledR} 12 \%$ Bis-Tris gels (Invitrogen) in MES SDS running buffer at $200 \mathrm{~V}$ for $35 \mathrm{~min}$. The molecular weight standard used was SeeBlue ${ }^{\circledR}$ Plus2 Pre-stained protein standard (Invitrogen). Proteins were stained using InstantBlue (Expedeon).

\section{Ultra-performance liquid chromatography mass spectrometry (UPLC-MS)}

Chromatographic separation of culture supernatants was carried out on an ACQUITY UPLC system (Waters) using an ACQUITY UPLC BEH $300 \AA \mathrm{C}$ - 18 column $(2.1 \mathrm{~mm}$ id $\times 100 \mathrm{~mm}$, particle size $1.7 \mu \mathrm{m})$ at $45^{\circ} \mathrm{C}$ with a flow rate of $0.250 \mathrm{~mL} / \mathrm{min}$. The mobile phase consisted of $0.1 \%$ formic acid in Milli-Q $\mathrm{H}_{2} \mathrm{O}$ (solvent A) and $0.1 \%$ formic acid in acetonitrile (solvent $\mathrm{B}$ ). The column was eluted using a gradient from 5\% B to $45 \%$ B over $4 \mathrm{~min}$ and $30 \mathrm{~s}$. The injection volume was $10 \mu \mathrm{L}$. UPLC column eluate was analysed by online electrospray ionization-time of flight mass spectrometry using a calibrated micrOTOF II (Bruker) mass spectrometer in positive ion mode. Data were analysed for relative quantitation using DataAnalysis 4.0 (Bruker) software by extracting the ion chromatogram (EIC) for the 722 mass-to-charge $(\mathrm{m} / \mathrm{z})$ analyte. Charge state deconvolution, to confirm the intact mass of the analyte, was performed using the maximum entropy method.

\section{Total DNA extraction}

Strains were grown at $30{ }^{\circ} \mathrm{C}$ with shaking $(200 \mathrm{rpm}$, $25 \mathrm{~mm}$ orbit) for approximately $24 \mathrm{~h}$ in $10 \mathrm{~mL}$ BMMD medium with and without the appropriate methionine concentration at $30{ }^{\circ} \mathrm{C}$ to an $\mathrm{OD}_{600}$ in the mid-log phase. Cells were harvested by centrifugation at $5000 \mathrm{~g}$ for $5 \mathrm{~min}$ and stored at $-80^{\circ} \mathrm{C}$. Total DNA was isolated using a Wizard ${ }^{\circledR}$ Genomic DNA isolation kit (Promega). DNA quantity was determined by $\mathrm{A}_{260}$ measurements and purity by $\mathrm{A}_{260} / \mathrm{A}_{280}$ ratio measurements using a NanoDrop 1000 UV-Vis spectrophotometer (Thermo Scientific).

\section{Total RNA extraction and CDNA synthesis}

Every $24 \mathrm{~h} 500 \mu \mathrm{L}$ culture samples were taken and cells harvested by centrifugation at $13,000 \mathrm{~g}$ for $2 \mathrm{~min}$. The pellets were resuspended in $300 \mu \mathrm{L}$ RNAlater (Qiagen) and stored at $4{ }^{\circ} \mathrm{C}$ for immediate stabilisation and protection of the RNA. Total RNA was extracted using the RNeasy Mini kit (Qiagen) with yeast spheroplasts 
prepared by lyticase (Sigma) digestion. To eliminate genomic DNA contamination, an additional DNase treatment was performed according to the RNeasy kit instruction with the RNase-free DNase set (Qiagen). Quantity of the extracted RNA was determined by $\mathrm{A}_{260}$ measurements and purity by $\mathrm{A}_{260} / \mathrm{A}_{280}$ ratio measurements using a NanoDrop $1000 \mathrm{UV}-\mathrm{Vis}$ spectrophotometer (Thermo Scientific). 100 ng DNase digested total RNA was used in a $20 \mu \mathrm{L}$ Superscript III (Invitrogen) reverse transcriptase reaction mixture according to the manufacturer's instructions.

\section{Quantitative real time polymerase chain reaction (RT-PCR)} TaqMan ${ }^{\circledR}$ primer/probe sequences were designed using Primer Express software (Applied Biosystems). Reactions were set up in total volumes of $25 \mu \mathrm{L}$ comprising $12.5 \mu \mathrm{L}$ of $2 \times$ TaqMan $^{\circledR}$ Gene Expression master mix (Applied Biosystems), $250 \mathrm{nM}$ probe, $1 \mu \mathrm{M}$ primers and $5 \mu \mathrm{L}$ of cDNA or DNA template (diluted 100-fold). The absence of genomic DNA contamination in RNA samples were checked by RT-PCR before cDNA synthesis using RNA as a template in RT-PCR (minus reverse transcriptase control). Blank samples (no template control) were incorporated in each assay. Reactions were carried out in triplicates and as singleplex reactions using an Applied Biosystems 7500 system with the thermocycling program consisting of one hold at $50{ }^{\circ} \mathrm{C}$ for $2 \mathrm{~min}$, another hold at $95{ }^{\circ} \mathrm{C}$ for $10 \mathrm{~min}$, followed by 40 cycles of $15 \mathrm{~s}$ at $95^{\circ} \mathrm{C}$ and $1 \mathrm{~min}$ at $60^{\circ} \mathrm{C}$. Transcript data were analysed using the relative standard curve method using TAF10 as endogenous control and gene copy-numbers were determined using FLP1 as the single-copy reference gene.

\section{Abbreviations \\ BMMD: basal minimal media dextrose; Ct: cycle threshold; ElC: extracted-ion chromatogram; hBD2: human $\beta$-defensin-2; HSA: human serum albumin; MES: 2-(N-morpholino) ethanesulfonic acid; rHA: recombinant human albumin; RT-PCR: reverse transcription polymerase chain reaction; SDS-PAGE: sodium dodecyl sulfate polyacrylamide gel electrophoresis; SFC: shake flask culture; UPLC-MS: ultra-performance liquid chromatography mass spectrometry.}

\section{Authors' contributions}

TSBM: Molecular biology, results analysis and preparation of manuscript. JH: Cloning and preliminary analysis. MJS: UPLC-MS. KB: Project supervision and results analysis. EP: Project supervision. SK: Project initiation and management. CJAF: Project management, supervision and preparation of manuscript. All authors read and approved the final manuscript.

\section{Author details}

${ }^{1}$ Novozymes Biopharma UK Limited, Castle Court, 59 Castle Boulevard, Nottingham NG7 1FD, UK. ${ }^{2}$ Department of Physics and Nanotechnology, Aalborg University, Skjernvej 4A, Aalborg East, 9220 Aalborg, Denmark.

\section{Acknowledgements}

This study was performed at Novozymes Biopharma UK Ltd during an international student placement from Aalborg University. We acknowledge the valuable contributions made by Ryan Hylands, Andrew Plumridge and Richard Woods.

\section{Competing interests}

Novozymes Biopharma UK Ltd (now Albumedix Ltd) authors declare competing interests as employees and/or inventors. EIP has no competing interests.

\section{Availability of data and materials}

The data that support the findings of this study are available from Albumedix Ltd, but restrictions apply to the availability of these data. Data are also available from the authors upon reasonable request and with permission of Albumedix Ltd and/or Novozymes A/S.

\section{Ethics approval and consent to participate}

Not applicable. The manuscript does not report data collected from humans or animals.

\section{Funding}

This study was funded by Novozymes Biopharma UK Ltd (now Albumedix Ltd).

Received: 11 September 2016 Accepted: 8 January 2017

Published online: 18 January 2017

\section{References}

1. Porro D, Gasser B, Fossati T, Maurer M, Branduardi P, Sauer M, Mattanovich D. Production of recombinant proteins and metabolites in yeasts: when are these systems better than bacterial production systems? Appl Microbiol Biotechnol. 2011;89:939-48.

2. Huang B, Guo J, Yi B, Yu X, Sun L, Chen W. Heterologous production of secondary metabolites as pharmaceuticals in Saccharomyces cerevisiae. Biotechnol Lett. 2008;30:1121-37.

3. Strausberg RL, Strausberg SL. Overview of protein expression in Saccharomyces cerevisiae. Curr Protoc Protein Sci 2001, Chapter 5: Unit 5-6.

4. Kaufmann A, Knop M. Genomic promoter replacement cassettes to alter gene expression in the yeast Saccharomyces cerevisiae. Strain Eng. 2011;765:275-94

5. Mizuguchi H, Hayakawa T. The tet-off system is more effective than the tet-on system for regulating transgene expression in a single adenovirus vector. J Gene Med. 2002;4:240-7.

6. Solow SP, Sengbusch J, Laird MW. Heterologous protein production from the inducible MET25 promoter in Saccharomyces cerevisiae. Biotechnol Prog. 2005;21:617-20

7. Thomas D, Surdin-Kerjan Y. Metabolism of sulfur amino acids in Saccharomyces cerevisiae. Microbiol Mol Biol Rev. 1997;61:503-32.

8. Sangsoda S, Cherest H, Surdin-Kerjan Y. The expression of the MET25 gene of Saccharomyces cerevisiae is regulated transcriptionally. Mol Gen Genet. 1985;200:407-14

9. Thomas D, Cherest H, Surdin-Kerjan Y. Elements involved in S-adenosylmethionine-mediated regulation of the Saccharomyces cerevisiae MET25 gene. Mol Cell Biol. 1989;9:3292-8.

10. Cherest H, Surdin-Kerjan Y, Antoniewski J, Robichon-Szulmajster H. S-adenosyl methionine-mediated repression of methionine biosynthetic enzymes in Saccharomyces cerevisiae. J Bacteriol. 1973;114:928-33.

11. Hansen J, Johannesen PF. Cysteine is essential for transcriptional regulation of the sulfur assimilation genes in Saccharomyces cerevisiae. Mol Gen Genet. 2000;263:535-42.

12. Mumberg D, Muller R, Funk M. Regulatable promoters of Saccharomyces cerevisiae: comparison of transcriptional activity and their use for heterologous expression. Nucleic Acids Res. 1994;22:5767-8.

13. Ardon O, Bussey H, Philpott C, Ward DMV, Davis-Kaplan S, Verroneau S, Jiang B, Kaplan J. Identification of a candida albicans ferrichrome transporter and its characterization by expression in Saccharomyces cerevisiae. J Biol Chem. 2001;276:43049.

14. Prein B, Natter K, Kohlwein SD. A novel strategy for constructing $\mathrm{N}$-terminal chromosomal fusions to green fluorescent protein in the yeast Saccharomyces cerevisiae. FEBS Lett. 2000;485:29-34.

15. Finnis CJ, Payne T, Hay J, Dodsworth N, Wilkinson D, Morton P, Saxton MJ, Tooth DJ, Evans RW, Goldenberg H, et al. High-level production of animalfree recombinant transferrin from Saccharomyces cerevisiae. Microb Cell Fact. 2010:9:87. 
16. Aerts AM, Francois IE, Cammue BP, Thevissen K. The mode of antifungal action of plant, insect and human defensins. Cell Mol Life Sci. 2008;65:2069-79.

17. Bauer F, Schweimer K, Kluver E, Conejo-Garcia JR, Forssmann WG, Rosch P, Adermann K, Sticht H. Structure determination of human and murine beta-defensins reveals structural conservation in the absence of significant sequence similarity. Protein Sci. 2001;10:2470-9.

18. Schroder JM, Harder J. Human beta-defensin-2. Int J Biochem Cell Biol. 1999;31:645-51

19. Schneider JJ, Unholzer A, Schaller M, Schafer-Korting M, Korting HC. Human defensins. J Mol Med (Berl). 2005;83:587-95.

20. De Smet K, Contreras R. Human antimicrobial peptides: defensins, cathelicidins and histatins. Biotechnol Lett. 2005;27:1337-47.

21. Joly S, Maze C, McCray PB Jr, Guthmiller JM. Human beta-defensins 2 and 3 demonstrate strain-selective activity against oral microorganisms. J Clin Microbiol. 2004;42:1024-9.

22. Brogden KA. Antimicrobial peptides: pore formers or metabolic inhibitors in bacteria? Nat Rev Microbiol. 2005;3:238-50.

23. Corrales-Garcia LL, Possani LD, Corzo G. Expression systems of human $\beta$-defensins: vectors, purification and biological activities. Amino Acids. 2011;40:5-13.

24. Vargues T, Morrison GJ, Seo ES, Clarke DJ, Fielder HL, Bennani J, Pathania U, Kilanowsk F, Dorin JR, Govan JRW, Mackay CL, Uhrin D, Campopiano DJ. Efficient production of human $\beta$-defensin 2 (HBD2) in Escherichia coli. Protein Pept Lett. 2009;16:668-76.

25. Xu Z, Peng L, Zhong Z, Fang X, Cen P. High-level expression of a soluble functional antimicrobial peptide, human $\beta$-defensin 2, in Escherichia coli. Biotechnol Prog. 2006;22:382-6.

26. Naik RR, Nebes $V$, Jones EW. Regulation of the proteinase $B$ structural gene PRB1 in Saccharomyces cerevisiae. J Bacteriol. 1997;179:1469-74.

27. Sleep D, Belfield GP, Ballance DJ, Steven J, Jones S, Evans LR, Moir PD, Goodey AR. Saccharomyces cerevisiae strains that overexpress heterologous proteins. Biotechnology (N Y). 1991;9:183-7.

28. Sleep D, Finnis C, Turner A, Evans L. Yeast 2 micron plasmid copy number is elevated by a mutation in the nuclear gene UBC4. Yeast. 2001;18:403-21.
29. Kerry-Williams SM, Gilbert SC, Evans LR, Ballance DJ. Disruption of the Saccharomyces cerevisiae YAP3 gene reduces the proteolytic degradation of secreted recombinant human albumin. Yeast. 1998;14:161-9.

30. Sleep D, Finnis C, Evans L. Enhanced protein expression through strain selection, gene disruption, improved vector design and co-expression of endogenous chaperones. Microb Cell Fact. 2006;5:S29.

31. Teste MA, Duquenne M, Francois JM, Parrou JL. Validation of reference genes for quantitative expression analysis by real-time RT-PCR in Saccharomyces cerevisiae. BMC Mol Biol. 2009;10:99.

32. Wenzel TJ, Teunissen AW, de Steensma HY. PDA1 mRNA: a standard for quantitation of mRNA in Saccharomyces cerevisiae superior to ACT1 mRNA. Nucleic Acids Res. 1995;23:883-4.

33. Futcher $A B$. The 2 micron circle plasmid of Saccharomyces cerevisiae. Yeast. 1988:4:27-40

34. Sutton A, Broach JR. Signals for transcription initiation and termination in the Saccharomyces cerevisiae plasmid 2 micron circle. Mol Cell Biol. 1985;5:2770-80.

35. Brønden A, Naver SV, Knop FK, Christensen M. Albiglutide for treating type 2 diabetes: an evaluation of pharmacokinetics/pharmacodynamics and clinical efficacy. Expert Opin Drug Metab Toxicol. 2015;11:1493-503.

36. Chinery SA, Hinchliffe E. A novel class of vector for yeast transformation. Curr Genet. 1989;16:21-5.

37. Orrweaver TL, Szostak JW. Yeast recombination - the association between double-strand gap repair and crossing-over. Proc Natl Acad Sci USA Biol Sci. 1983;80:4417-21.

38. Ma H, Kunes S, Schatz PJ, Botstein D. Plasmid construction by homologous recombination in yeast. Gene. 1987;58:201-16.

39. Payne T, Finnis C, Evans LR, Mead DJ, Avery SV, Archer DB, Sleep D. Modulation of chaperone gene expression in mutagenized Saccharomyces cerevisiae strains developed for recombinant human albumin production results in increased production of multiple heterologous proteins. Appl Environ Microbiol. 2008;74:7759-66.

\section{Submit your next manuscript to BioMed Central and we will help you at every step:}

- We accept pre-submission inquiries

- Our selector tool helps you to find the most relevant journal

- We provide round the clock customer support

- Convenient online submission

- Thorough peer review

- Inclusion in PubMed and all major indexing services

- Maximum visibility for your research

Submit your manuscript at www.biomedcentral.com/submit
() Biomed Central 